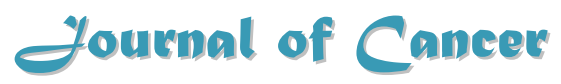

$2011 ; 2: 52-62$

Research Paper

(C) Ivyspring International Publisher. All rights reserved

\title{
Smoking and Prognostic Factors in an Observational Setting in Patients with Advanced Non-Small Cell Lung Carcinoma
}

\author{
Chien-Te Li ${ }^{1}$ Magdalena Marek², Salih Z Guclu³, Younseup Kim4, Mohamed Meshref ${ }^{5}$, Shukui Qin ${ }^{6}$, Zbig- \\ niew Kadziola7, Kurt Krejcy ${ }^{7}$, Sedat Altug ${ }^{8}{ }^{\circledR}$
}

1. Chest Department, Changhua Christian Hospital, Changhua City, and the Institute of Medical and Molecular Toxicology, Chung Shan Medical University, Taichung City, Taiwan.

2. Department of Lung Cancer Chemotherapy, Bystra Hospital, Bystra, Poland.

3. Department of Chest Diseases, Izmir Chest Diseases Research Hospital, Izmir, Turkey.

4. Department of Pulmonology, Dankook University Hospital, Cheonan Choong Nam, Republic of Korea.

5. Department of Clinical Oncology, Faculty of Medicine, Cairo University, Cairo, and Eli Lilly, Egypt.

6. Chinese PLA Cancer Center, Nanjing Bayi Hospital, Nanjing, China.

7. Eli Lilly Regional Operations, Vienna, Austria.

8. Medical Department, Eli Lilly Turkey, Istanbul, Turkey.

Corresponding author: Sedat Altug, MD, Medical Department, Eli Lilly Turkey, Istanbul, Turkey. altug_sedat@lilly.com.

Received: 2010.11.18; Accepted: 2011.01.06; Published: 2011.01.11

\begin{abstract}
Background: This prospective observational study estimated the effect of prognostic factors, particularly continued smoking during therapy, on survival in advanced non-small cell lung cancer (NSCLC) patients receiving gemcitabine-platinum. Further, prognostic factors were used to build a survival model to improve prognosis prediction in naturalistic clinical settings.

Methods: Eligibility criteria included: Stage IIIB/IV NSCLC, no prior chemotherapy, and Eastern Cooperative Oncology Group (ECOG) performance status 0 or I. A Cox regression model was constructed and validated by randomizing patients into two datasets (Construction [C]:Validation [V]; 3:I ratio). Country, disease stage, hypercalcemia, " $N$ " factor, weight reduction, performance status, and superior vena cava obstruction were pre-defined variables forced into the model. Continued smoking was tested with adjustment for these variables.

Results: One thousand two hundred and fourteen patients $(C=89 I$ and $V=323)$ were enrolled. The final predictive model, established in the Construction dataset, identified four significant $(p \leq 0.05)$ and independent predictors of survival, which were disease stage, performance status, gemcitabine-platinum regimen, and T-stage. Smoking during therapy was not significantly associated with survival (Hazard Ratio [95\% Cl]: 0.955 [0.572, I.596], $\mathrm{p}=0.86$ । 8; versus never smokers).

Conclusions: Although continued smoking during therapy was not significantly associated with shorter survival, the model developed in this study forms an evidence-based approach to assessing prognosis in advanced stage NSCLC.
\end{abstract}

Key words: smoking; observational; NSCLC; prognostic factors; predictive modeling.

\section{Background}

Lung cancer is one of the leading causes of cancer-related deaths worldwide [1-2], with non-small cell lung cancer (NSCLC) representing approximately $75 \%-85 \%$ of all types of lung cancer [3]. In the majority of cases, patients with NSCLC present with locally advanced (Stage III) or metastatic disease (Stage IV) [4].

Smoking is the single most important cause of NSCLC [5-8], with approximately $85 \%$ of human lung cancers arising in current or former smokers. No 
prospective study has been published that evaluated the effect of smoking on survival in advanced NSCLC treated with chemotherapy. Furthermore, limited data is available on the effect of smoking on chemotherapy toxicity. Considering the high incidence of advanced stage NSCLC and the common use of chemotherapy in these patients, these questions appear to be of major clinical relevance.

Numerous factors have been shown to influence survival and toxicity in patients with advanced NSCLC, such as disease stage, performance status, smoking, age, weight loss, and gender [5,9-12]. Additionally, molecular markers such as $p 53$ and ras mutations, and expression of ERCC1, beta-tubulin III and RRM1, have been found to influence treatment outcome [5,13-15].

The main aim of this prospective, observational study was to estimate the effect of prognostic factors, in particular, continued smoking during therapy, on survival in patients with advanced NSCLC receiving gemcitabine-platinum as first-line therapy. Further, prognostic factors identified in previous studies were used to build a survival model with the aim of improving prognosis prediction, in naturalistic clinical settings, in patients with advanced NSCLC who are receiving gemcitabine-platinum as first-line therapy.

\section{Methods}

\section{Study Design}

This prospective, non-interventional, international, observational study (B9E-AA-B004) was designed to estimate the effect of prognostic factors, including continued smoking during therapy, on treatment outcomes in patients with advanced NSCLC receiving gemcitabine-platinum as first-line therapy as part of their routine care. To ensure this study reflected real-life clinical practice, all care provided to the patients (including visit frequency, procedures performed at visits, and advice regarding smoking behavior) was at the discretion of the participating oncologist. Patients were recruited between June 2004 and October 2005 from nine countries (China, Egypt, Israel, Pakistan, Poland, Romania, South Korea, Taiwan, and Turkey) and were followed for survival until death, 18 months after the start of treatment, or lost to follow-up. The study was conducted in accordance with the ethics and regulatory requirements of each country and all participants provided written informed consent prior to enrollment in the study.

\section{Participants}

Patients were eligible if they: (a) were diagnosed with Stage IIIB or IV NSCLC; (b) were chemonaive; (c) received gemcitabine in combination with platinum (carboplatin or cisplatin) as part of their routine care; (d) had an Eastern Cooperative Oncology Group (ECOG) performance status $\leq 1$; and (e) not simultaneously participating in a gemcitabine-platinum interventional study. The dosing schedule of gemcitabine-platinum therapy, use of concomitant medications, supportive care measures, and all subsequent lines of tumor therapy were at the discretion of the treating oncologist.

\section{Effectiveness and Safety Measures}

Eligible participants who received at least one dose of gemcitabine-platinum were evaluated for effectiveness and safety. Effectiveness was measured by survival, defined as the time from start of gemcitabine-platinum therapy to the date of death due to any cause. The effect of prognostic factors on an occurrence of any selected adverse events (AEs; neutropenia, thrombocytopenia, reduced haemoglobin, infection requiring hospitalisation or intravenous antibiotics, respiratory distress syndrome, dyspnea, death, or life-threatening toxicity) and methylation of $p 16$ and RASSF1A were assessed.

\section{Statistical Analyses}

Statistical analyses were performed using the SAS program (Version 9, SAS Institute, Cary, NC). A total of 3000 patients were planned to be enrolled with patients allocated in a 3:1 ratio to the Construction:Validation datasets to provide $80 \%$ power to draw the conclusion that continued smoking during chemotherapy reduces median survival time by $15 \%$ after balancing for clinical characteristics that have a statistically significant effect on survival. To identify potential prognostic factors associated with survival in advanced NSCLC patients treated with first-line gemcitabine-platinum, a total of 42 prognostic factors were analyzed. Some of the factors analyzed in this study were previously identified as potential prognostic factors in NSCLC [17] and include: continued smoking during therapy; number of cigarettes/day during therapy; baseline smoking level; continuing smoker versus ex-smoker; heavy smoker at baseline; never smoked; race; country; disease stage; hypercalcemia; TNM staging (T, N, and M); weight loss $>10 \%$; performance status; superior vena cava obstruction present; age $<70$ years; gemcitabine-platinum regimen; largest tumor $>5 \mathrm{~cm}$; gender; metastatic disease (extra-thoracic; liver; bone; brain); diagnosis (histology); dyspnea present; cough present; hemoptysis present; pain present; expectoration present; chronic obstructive pulmonary disease present; pleural effusion present; $p 16$ status; RASSF1A status; albumin 
(normal range indicator); hemoglobin; aspartate aminotransferase (AST/SGOT); alanine aminotransferase (ALT/SGPT); bilirubin (total); albumin; lactic dehydrogenase; calcium. Univariate Cox regression analysis was used to first assess the association between each variable and survival, followed by multivariate stepwise Cox regression analysis for variable selection (with entry and stay cutoff levels of 0.1). If information on any baseline or treatment variable was missing in $>10 \%$ of patients, that variable was not used to build the primary model. Results are reported as hazard ratios (HR) with $95 \%$ confidence intervals.

Additional sensitivity analyses were performed to examine the potential impact of missing data. This involved effect of smoking variables adjusted in multivariate models, also for variables where $>10 \%$ of the data was missing using the same model selection process described above.

Validation of the final predictive model was assessed using a Cox regression on the Validation dataset with the values of the linear predictor calculated from the coefficients estimated in the Construction dataset [16]. Also, predicted one-year survival was directly compared with the actual one-year survival for patients in the Validation dataset.

The occurrence of AEs and association of $p 16$ and RASSF1A methylation to baseline prognostic factors was analyzed using a univariate and multivariate (stepwise) logistic regression.

\section{Results \\ Participant Characteristics and Treatments}

Baseline clinical and demographic characteristics, including potentially important prognostic factors, are outlined in Table 1. Of 1214 patients enrolled from nine countries/regions, 891 (73.4\%) were assigned to the Construction dataset and $323(26.6 \%)$ to the Validation dataset. Patients were on average 60.5 years of age, with $75.1 \%$ being male. Approximately half of the patients had NSCLC of adenocarcinoma origin $(48.9 \%)$, while approximately two-thirds of patients $(69.4 \%)$ were assigned to gemcitabine-cisplatin treatment. The mean number of gemcitabine-platinum cycles received was 3.76 (95\% CI: $3.66,3.86)$, with $34.1 \%$ of patients receiving no therapy post gemcitabine-platinum treatment. Post gemcitabine-platinum treatment therapies are outlined in Table 2, with the most common second-line treatment approaches being docetaxel $(18.3 \%)$ or radiotherapy (17.1\%). Of the 1214 patients enrolled, $253(20.8 \%)$ were alive at study completion, 637 (52.5\%) had died, $310(25.5 \%)$ were lost to follow-up, while data was not available for $14(1.2 \%)$. Three hundred and nineteen $(26.3 \%)$ patients discontinued study therapy due to inadequate response, while $48(4.0 \%)$ discontinued study therapy due to AEs.

Originally, 3000 patients were planned to be enrolled in this study; however, based on slower than expected recruitment, a decision was made to stop accrual after 17 months. At the time, a total of 1214 qualified patients had been enrolled in this study.

Table I. Baseline Characteristics of Potential Prognostic Factors (Construction and Validation Datasets)

\begin{tabular}{|c|c|c|c|}
\hline Characteristic & $\begin{array}{l}\text { Construction Dataset } \\
\qquad \mathrm{N}=891\end{array}$ & $\begin{array}{c}\text { Validation Dataset } \\
\quad \mathrm{N}=323\end{array}$ & $\begin{array}{c}\text { Total } \\
\mathbf{N}=1214\end{array}$ \\
\hline \multicolumn{4}{|l|}{ Country of Treatment, $n(\%)$} \\
\hline China & $208 \quad(23.3)$ & $(28.5)$ & $(24.7)$ \\
\hline Egypt & $158 \quad(17.7)$ & $(13.0)$ & $(16.5)$ \\
\hline Israel & $19 \quad(2.1)$ & $(6.2)$ & $39 \quad(3.2)$ \\
\hline Pakistan & $(7.2)$ & $(4.0)$ & $(6.3)$ \\
\hline Poland+Romania & $(5.6)$ & (13.6) & (7.7) \\
\hline South Korea & $147 \quad(16.5)$ & $(14.2)$ & (15.9) \\
\hline Taiwan & $179 \quad(20.1)$ & $(5.0)$ & (16.1) \\
\hline Turkey & $66 \quad(7.4)$ & $(15.5)$ & $(9.6)$ \\
\hline \multicolumn{4}{|l|}{ Performance Status (ECOG), n(\%) } \\
\hline 0 & $294 \quad(33.0)$ & $(35.6)$ & $(33.7)$ \\
\hline 1 & $596 \quad(66.9)$ & $208 \quad(64.4)$ & $804 \quad(66.2)$ \\
\hline Missing data & $(0.1)$ & - & $(0.1)$ \\
\hline \multicolumn{4}{|l|}{ Tumor Stage, $\mathbf{n}(\%)$} \\
\hline Stage IIIB & $385 \quad(43.2)$ & $129 \quad(39.9)$ & $(42.3)$ \\
\hline Stage IV & $501 \quad(56.2)$ & $191 \quad(59.1)$ & $692 \quad(57.0)$ \\
\hline Missing data & $5 \quad(0.6)$ & $3 \quad(0.9)$ & $8 \quad(0.7)$ \\
\hline \multicolumn{4}{|l|}{ Age, years } \\
\hline Mean (SD) & $61.0(10.8)$ & $59.2(10.6)$ & $60.5(10.8)$ \\
\hline Missing data & $9(1.0)$ & $4(1.2)$ & $13(1.1)$ \\
\hline \multicolumn{4}{|l|}{ Gender, $\mathbf{n}(\%)$} \\
\hline Female & $222 \quad(24.9)$ & $(24.1)$ & $(24.7)$ \\
\hline
\end{tabular}




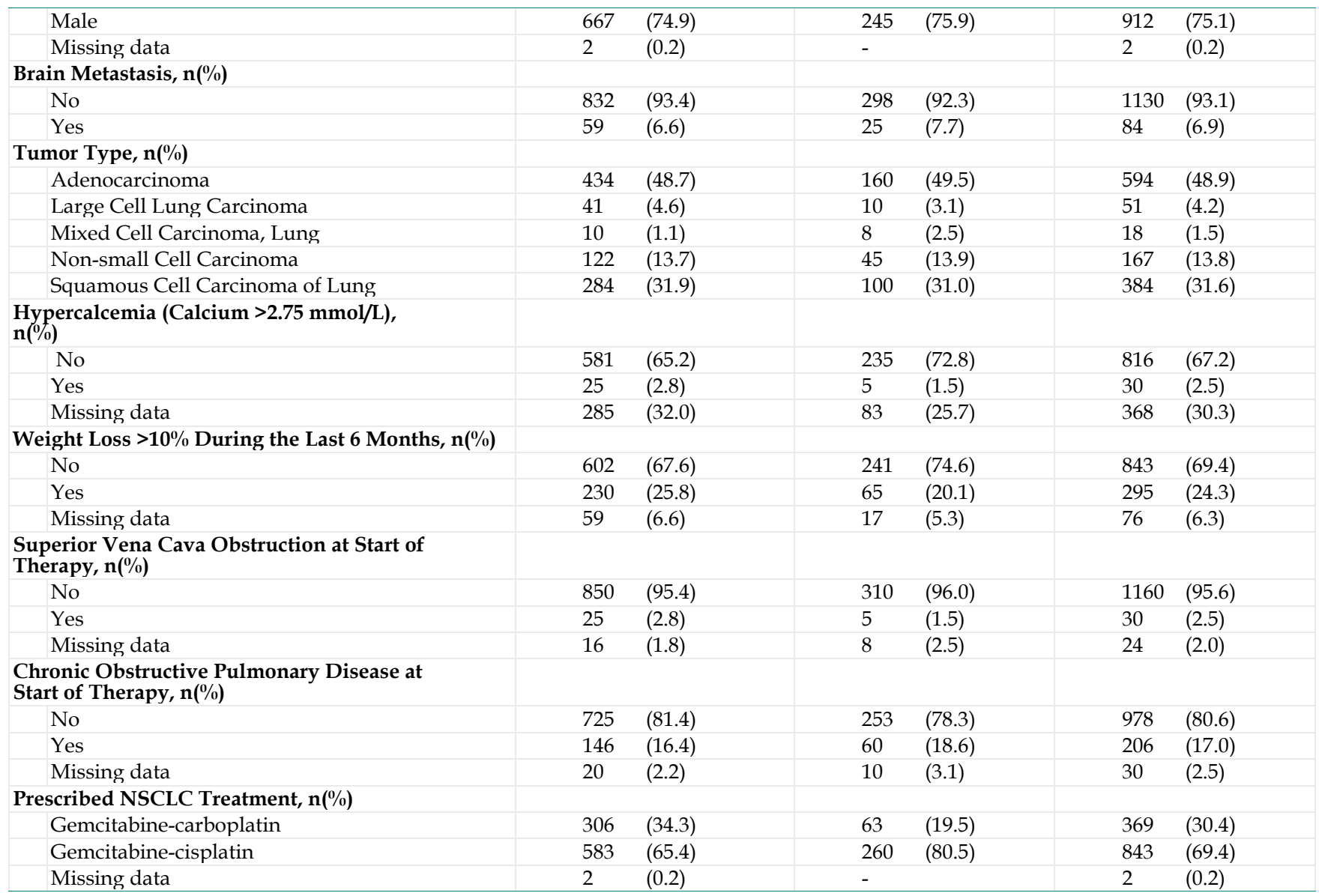

Abbreviations: ECOG = Eastern Cooperative Oncology Group; $\mathrm{N}=$ total number of patients; $\mathrm{n}=$ number of patient in specified category; NSCLC $=$ non-small cell lung cancer; $\mathrm{SD}=$ standard deviation

Table 2. Post Gemcitabine-Platinum Treatment Approaches

\begin{tabular}{|c|c|c|c|}
\hline Post Gemcitabine-Platinum Therapy, n(\%) & $\begin{array}{l}\text { Construction Dataset } \\
\qquad \mathrm{N}=891\end{array}$ & $\begin{array}{l}\text { Validation Dataset } \\
\qquad=323\end{array}$ & $\begin{array}{c}\text { Total } \\
\mathrm{N}=1214\end{array}$ \\
\hline Died on Gemcitabine-Platinum Therapy & $(10.8)$ & $18(5.6)$ & $114 \quad(9.4)$ \\
\hline No Post Gemcitabine-Platinum Therapy & $(34.7)$ & $105 \quad(32.5)$ & $(34.1)$ \\
\hline \multicolumn{4}{|l|}{ Post Gemcitabine-Platinum Therapy ${ }^{\top}$} \\
\hline Docetaxel & $(19.0)$ & $(16.4)$ & (18.3) \\
\hline Paclitaxel & $57(6.4)$ & $11(3.4)$ & $68(5.6)$ \\
\hline Vinorelbine & $39(4.4)$ & $14(4.3)$ & $53(4.4)$ \\
\hline Gefitinib & $59(6.6)$ & $29(9.0)$ & $88(7.2)$ \\
\hline Radiotherapy & $140 \quad(15.7)$ & $67 \quad(20.7)$ & $207 \quad(17.1)$ \\
\hline Other & $(10.7)$ & $(10.5)$ & $(10.6)$ \\
\hline
\end{tabular}

* Patients may have received more than one post gemcitabine-platinum therapy.

\section{Effectiveness and Toxicity}

The median overall survival time was 12.7 months (95\% CI: 11.5, 13.7; $\mathrm{n}=1213)$. On completion of study therapy, of the 1214 patients enrolled, 594
$(48.9 \%)$ had no reported progression of disease. Of the $637(52.5 \%)$ that died during the study, the majority $(95.6 \%)$, in the opinion of the investigator, died as a result of study disease. 
Of the 1214 patients enrolled, 266 (21.9\%) reported at least one AE. The most frequently reported AEs were low hemoglobin count $(<8 \mathrm{~g} / \mathrm{dL} ; \mathrm{n}=161$, $13.3 \%)$, low neutrophil count $\left(<1.0 \times 10^{9} / \mathrm{L}\right.$ associated with fever of $\geq 38.5^{\circ} \mathrm{C}$ or documented infection; $\mathrm{n}=99$, $8.2 \%)$, and thrombocytopenia $\left(<50 \times 10^{9} / \mathrm{L}\right.$ with bleeding; $\mathrm{n}=73,6.0 \%$ ).

\section{Univariate Analyses of Survival}

Of 42 prognostic factors analyzed, 16 were found by univariate Cox regression analysis to be significantly $(\mathrm{p} \leq 0.05)$ associated with survival, including baseline smoking level; heavy smoker at baseline; race; country; disease stage; performance status; superior vena cava obstruction; age $<70$ years; TNM staging; largest tumor $>5 \mathrm{~cm}$; gender; metastatic liver, bone and brain disease; dyspnea present; chronic obstructive pulmonary disease; albumin (normal range indicator); and lactic dehydrogenase.

\section{Development and Validation of the Predictive Model for Survival}

Firstly, all variables (excluding smoking variables) with $<10 \%$ missing values were included in the initial Cox's model. A stepwise Cox regression was then performed with essential factors identified by Brundage et al (2002) [17]. Country, disease stage, hypercalcemia, "N" factor, weight reduction, performance status, and superior vena cava obstruction were forced into the model. As a result, 6 variables were additionally selected by the regression (gemcitabine-platinum regimen, T-stage, chronic obstructive pulmonary disease, metastatic brain disease, gender, and diagnosis). The final predictive model (Figure 1) identified four significant $(\mathrm{p} \leq 0.05)$ and independent predictors of survival, which were disease stage, performance status, gemcitabine-platinum regimen, and T-stage. No smoking variables were represented in the final predictive model.

Cox regression performed on the Validation dataset showed high significance $(p=0.0008)$ of linear predictor calculated using the coefficients estimated in the Construction dataset. Patients in the Validation dataset $(n=323)$ were also classified into three groups based on the predicted one-year survival probabilities forecast from the final predictive model: (1) predicted probability $<0.2$; (2) predicted probability ranging from $\geq 0.2$ to $<0.5$; and (3) predicted probability $\geq 0.5$. The predicted and observed proportions surviving were calculated for each of the above groups (Figure
2). These analyses support the predictive model being strongly associated with actual survival in the Validation dataset.

\section{Development of the Predictive Model for Ad- verse Events}

Of 42 prognostic factors analyzed, 6 were found by univariate logistic regression analysis to be significantly $(p \leq 0.05)$ associated with AEs, which were country, gemcitabine-platinum regimen, largest tumor $>5 \mathrm{~cm}$, presence of hemoptysis, presence of pain, and albumin.

The final predictive model, as established in the Construction dataset using multivariate stepwise logistic regression (with gender forced into the model, and excluding smoking variables from the selection process), identified 5 significant $(p \leq 0.05)$ and independent predictors of AEs, that being disease stage (IIIB versus IV), country, weight loss $>10 \%$, age $<70$ years, and the presence of pain. The smoking variables added to the established model did not reach significance $(p>0.05)$.

\section{Effect of Continued Smoking during Therapy on Survival and Adverse Events}

Smoking characteristics at baseline and during the study are outlined in Table 3 . Overall, $70.8 \%$ of patients had smoked at some point prior to therapy (i.e. ever smokers). Approximately half of ever smokers $(53.7 \%)$ had ceased smoking at initiation of therapy or within 6 months prior to treatment start, while $11.2 \%$ of smokers continued to smoke during therapy. Of those patients continuing to smoke during therapy, the mean number of cigarettes smoked per day was 16.57 (95\% CI: 13.0, 20.1). There was no statistically significant difference in survival observed between "never smokers" and "ever smokers", with an unadjusted HR (versus never smokers) of 1.143 (95\% CI: 0.925, 1.441; $p=0.2155)$. None of the smoking variables forced into the established multivariate model were significantly associated with survival, with an adjusted HR of 0.955 (95\% CI: 0.572, 1.596) observed for continued smoking during therapy (versus never smokers; $\mathrm{p}=0.8618$ ), and an adjusted HR of 0.905 (95\% CI: $0.648,1.263$ ) observed for ex-smokers (versus never smokers; $\mathrm{p}=0.5579$ ). No statistically significant association was observed between AEs and continued smoking during therapy (adjusted OR=1.297 [95\% CI: 0.716, 2.350]; $\mathrm{p}=0.3912$ ). 


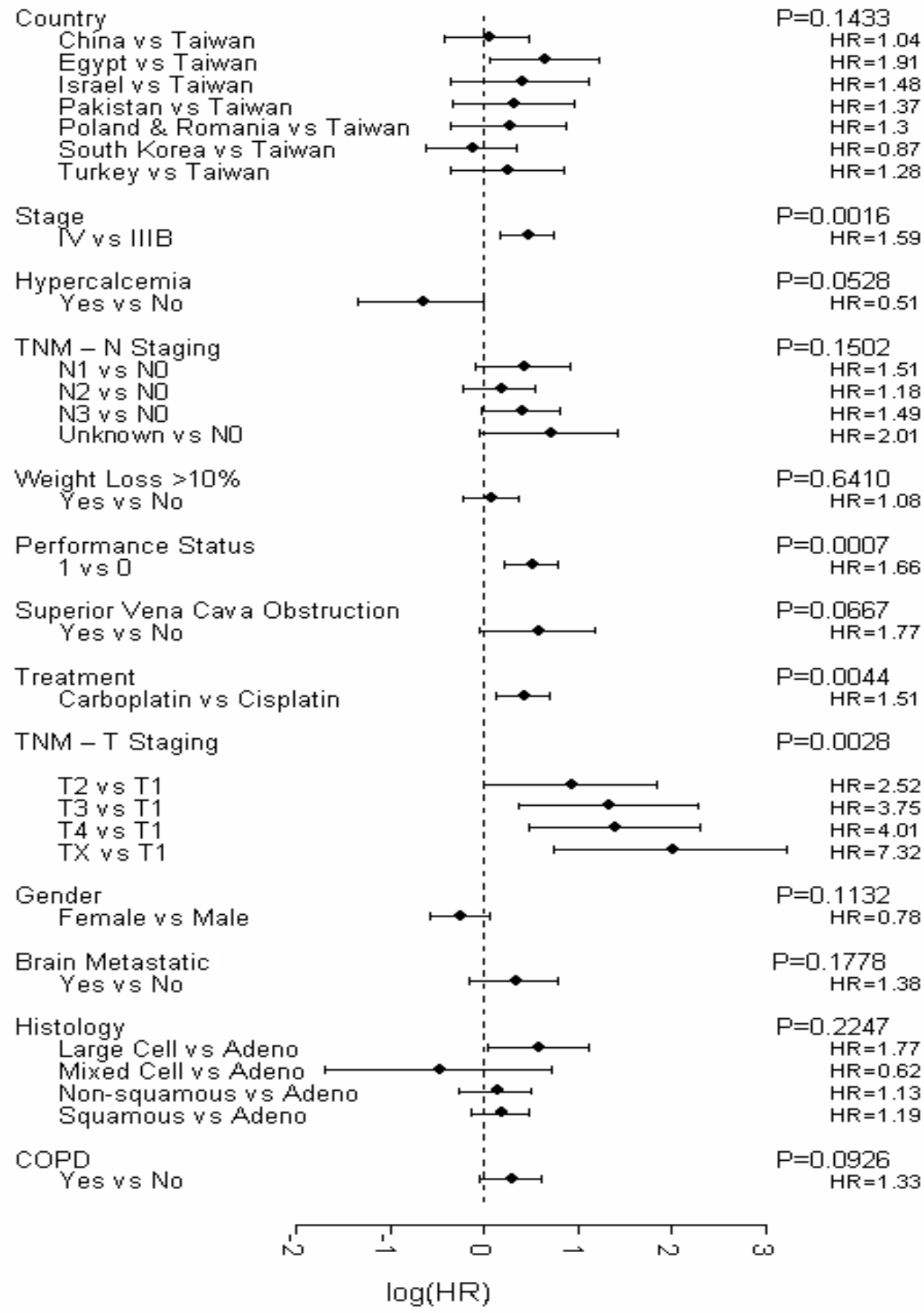

Figure I. Final Predictive Model for Survival (Construction Dataset). Abbreviations: adeno = adenocarcinoma; COPD = chronic obstructive pulmonary disease; $\mathrm{HR}=$ hazard ratio; TNM = tumor, node, metastasis; vs = versus. 


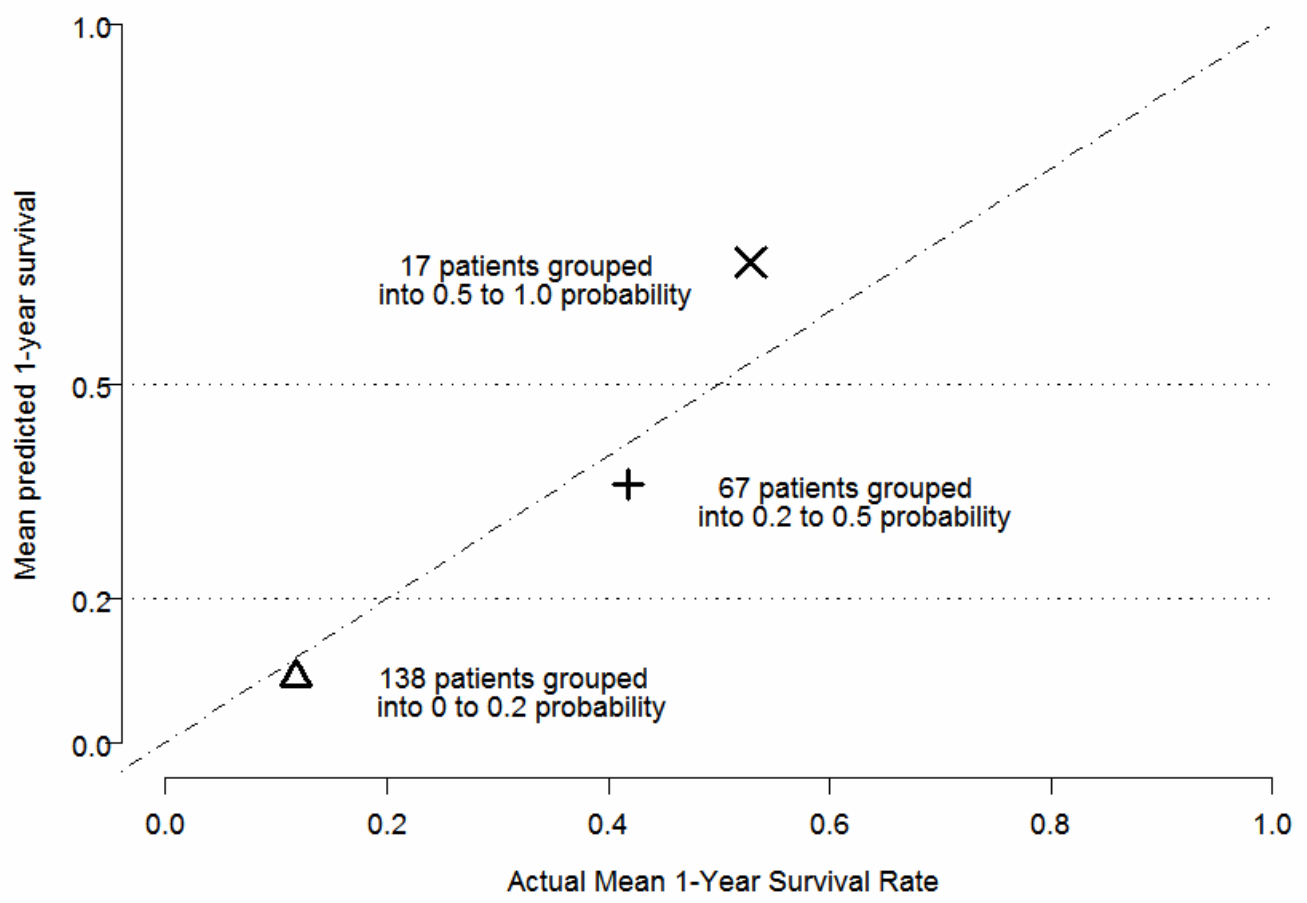

Figure 2. Validation of the Predictive Model: Predicted versus Actual One-Year Survival (Validation Dataset).

Table 3. Smoking Characteristics at Baseline and During Study (Construction and Validation Datasets)

\begin{tabular}{|c|c|c|c|}
\hline Smoking Characteristic, $\mathrm{n}(\%)$ & $\begin{array}{l}\text { Construction Dataset } \\
\mathrm{N}=891\end{array}$ & $\begin{array}{l}\text { Validation Dataset } \\
\mathrm{N}=323\end{array}$ & $\begin{array}{l}\text { Total } \\
\mathrm{N}=1214\end{array}$ \\
\hline \multicolumn{4}{|l|}{ Ever Smoked Prior to Therapy } \\
\hline No & $224(25.1)$ & $88(27.2)$ & $312(25.7)$ \\
\hline Yes & 629 (70.6) & $230(71.2)$ & $859(70.8)$ \\
\hline Missing data & $38(4.3)$ & $5(1.5)$ & $43(3.5)$ \\
\hline \multicolumn{4}{|l|}{ Pack-Years Smoked Prior to Therapy } \\
\hline $\mathrm{n}$ & 620 & 229 & 849 \\
\hline Mean $(95 \%$ CI) & $45.06(42.6,47.6)$ & $49.71(44.5,54.9)$ & $46.31(44.0,48.6)$ \\
\hline \multicolumn{4}{|c|}{$\begin{array}{l}\text { Time from Smoking Cessation to } \\
\text { Treatment Start for Patients who Ever Smoked Prior to Therapy } †\end{array}$} \\
\hline No cessation & $47(7.5)$ & $32(13.9)$ & $79(9.2)$ \\
\hline Restarted after cessation & $36(5.7)$ & $20(8.7)$ & $56(6.5)$ \\
\hline 0 (cessation at Treatment Start) ${ }^{\top}$ & $46(7.3)$ & $20(8.7)$ & $66(7.7)$ \\
\hline$>0 \&<=1 \mathrm{mo}$ & $129(20.5)$ & $51(22.2)$ & $180(21.0)$ \\
\hline$>1 \mathrm{mo} \mathrm{\&}<=6 \mathrm{mo}$ & $168(26.7)$ & 47 (20.4) & $215(25.0)$ \\
\hline$>6 \mathrm{mo} \&<=1 \mathrm{yr}$ & $38(6.0)$ & $1(0.4)$ & $39(4.5)$ \\
\hline$>1 \mathrm{yr} \&<=5 \mathrm{yr}$ & 75 (11.9) & $29(12.6)$ & $104(12.1)$ \\
\hline$>5 \mathrm{yr} \&<=10 \mathrm{yr}$ & $30(4.8)$ & $10(4.3)$ & $40(4.7)$ \\
\hline$>10 y r$ & $60(9.5)$ & $20(8.7)$ & $80(9.3)$ \\
\hline Mean $(95 \%$ CI), years $\ddagger$ & $2.96(2.38,3.53)$ & $3.33(2.17,4.48)$ & $3.05(2.53,3.57)$ \\
\hline \multicolumn{4}{|l|}{ Smoking During Therapy§ } \\
\hline No & $801(89.9)$ & $269(83.3)$ & $1070(88.1)$ \\
\hline Yes \# & $83(9.3)$ & $53(16.4)$ & $136(11.2)$ \\
\hline Missing data & $7(0.8)$ & $1(0.3)$ & $8(0.7)$ \\
\hline \multicolumn{4}{|l|}{ Cigarettes Per Day During Therapy } \\
\hline $\mathrm{n}$ & 83 & 53 & 136 \\
\hline Mean $(95 \%$ CI $)$ & $15.05(10.6,19.5)$ & $18.94(12.9,25.0)$ & $16.57(13.0,20.1)$ \\
\hline \multicolumn{4}{|l|}{$\begin{array}{l}\text { Heavy Smoker as a Proportion of All } \\
\text { Patients }\end{array}$} \\
\hline No & $397(44.6)$ & $155(48.0)$ & $552(45.5)$ \\
\hline
\end{tabular}




\begin{tabular}{|c|c|c|c|}
\hline Yes & 447 (50.2) & $162(50.2)$ & $609(50.2)$ \\
\hline Missing data & $47(5.3)$ & $6(1.9)$ & $53(4.4)$ \\
\hline
\end{tabular}

† Patients who stopped smoking but without giving a stop date should be considered to have stopped just before the start of therapy.

¥ Patients who stopped \& restarted are not included.

$\S$ Patients who have the cessation date > therapy start date and their 'No. cigarettes during therapy' is entered as zero or blank are considered not smoking during therapy.

\# One patient, who had never smoked prior to therapy, started smoking during therapy.

\section{Association of $p / 6$ and RASSFIA Methylation Status to Baseline Prognostic Factors and Ad- verse Events}

Methylation status of $p 16$ and RASSF1A was analyzed in 86 patients, with hypermethylation observed in $15(17.4 \%, p 16)$ and $8(9.3 \%$, RASSF1A) patients, respectively. Thirty-nine prognostic factors were analyzed by univariate and multivariate (stepwise) logistic regression. In those with RASSF1A hypermethylation, no factors were identified as significant following univariate $(\mathrm{n}=86)$ and stepwise logistic regression $(n=65)$. In those with $p 16$ hypermethylation, the following factors were found to be significant in univariate analyses $(n=86)$ : performance status ( 1 versus 0 , unadjusted $\mathrm{OR}=10.240$ [95\% CI: $1.276,82.162] \mathrm{p}=0.0286)$, extra-thoracic metastatic disease (yes versus no, unadjusted OR=6.891 [95\% CI: $1.968,24.124], \mathrm{p}=0.0025)$, and total bilirubin $(1 \mu \mathrm{mol} / \mathrm{L}$ versus 0 , unadjusted $\mathrm{OR}=1.240$ [95\% CI: 1.037, 1.482], $\mathrm{p}=0.0181)$. Following stepwise regression $(\mathrm{n}=65)$, extra-thoracic metastatic disease was identified as a significant factor (yes versus no, OR=5.595 [95\% CI: 1.342, 23.333], $\mathrm{p}<0.0181$ ).

Neither $p 16$ nor RASSF1A were significant when added to the established predictive model for AEs. Additionally, the univariate logistic regression did not identify methylation factors as predictive of AEs.

\section{Discussion}

In this prospective, observational study, the effect of prognostic factors, in particular continued smoking during therapy, on survival and other treatment outcomes, was assessed in patients with advanced NSCLC receiving gemcitabine-platinum as first-line therapy. Continued smoking during gemcitabine-platinum therapy was not associated with shorter survival in patients with advanced NSCLC. Construction and validation of a predictive model identified four independent prognostic factors associated with survival: disease stage, performance status, gemcitabine-platinum regimen, and T-stage.

The greatest risk factor associated with lung cancer is cigarette smoking [18-19], with approximately $85 \%$ of all lung cancer cases in men and $47 \%$ in women attributed to tobacco smoking [1]. Previous studies have identified an association towards longer survival [19], as well as statistically significant differences in survival [20], between "never smokers" and "ever smokers" who have undergone chemotherapy. Our study had a similar proportion of never smokers $(25.7 \%)$ compared to previous studies $(14.5 \%$ [21]; $16 \%$ [20]; 36.3\% [19]). However, unlike these other studies, there was no statistically significant difference in survival observed between "never smokers" and "ever smokers" receiving gemcitabine-platinum as first-line therapy. A retrospective study by Nguyen et al (2006) in which exploratory subgroup analyses were performed, reported similar findings to this study, in which no significant difference in median survival time between "ever smokers" and "never smokers" was observed in patients receiving gemcitabine-cisplatin therapy [22]. However, gemcitabine-cisplatin was also a therapy utilized in the study by Scagliotti et al (2008) and an association towards longer survival was observed in "never smokers" (15.3 months) compared to "ever smokers" (10.3 months) [21]. In terms of continued smoking during therapy, in our study, fewer patients (11.2\%) continued smoking during therapy when compared to a previous study (48\%) [20]. It is interesting to note that the retrospective study of Tsao et al (2006) encompassed patients treated with first-line chemotherapy between 1993 and 2002 [20], while our study reflects patient treatment during 2004-2005. It is therefore possible that the lower proportion of patients continuing to smoke during therapy in our study reflects the increased influence that oncologists are having on their patients as well as a greater public awareness of the association between smoking and lung cancer. Despite differences in the proportion of patients continuing smoking during therapy, similar findings in terms of the impact of continued smoking during therapy on survival were noted between our study and Tsao et al (2006), with no statistically significant difference in survival observed between those patients continuing to smoke during therapy and those who discontinued prior to therapy initiation [20]. This suggests the detrimental effects of cigarette 
smoking may occur earlier in disease progression. Another explanation may be that the effects of continued smoking during therapy are not evident in patients treated with chemotherapy in late stage disease as their duration of survival is limited. Additionally, bias as a result of the non-randomized, unblinded design of the study and confounding are inherently associated with observational studies and should be considered when interpreting the results. The effect of continued smoking during therapy on toxicity was also analyzed in this study. Although an association towards a greater rate of AEs was observed in patients who continued to smoke during therapy, this was not statistically significant.

In addition to smoking, numerous factors have been shown to influence survival and toxicity in patients with advanced NSCLC, such as disease stage, performance status, age, weight loss, and gender [5,9-12,19-20]. In this study, development of a predictive model in a Construction dataset, and subsequent validation with a Validation dataset, led to the identification of four significant $(p \leq 0.05)$ and independent predictors of survival, which were disease stage (IV versus IIIB), performance status (ECOG 1 versus 0 ), gemcitabine-platinum regimen (carboplatin versus cisplatin), and T-stage (versus T1). Performance status has been shown in a number of trials to be a powerful predictor of survival [9,11,19-20,23-25]. Similarly, in this study, performance status was identified as an independent prognostic factor, with improved survival associated with a better baseline performance status. In this study, disease stage was also identified as an independent prognostic factor, with improved survival noted in those patients with Grade IIIB NSCLC when compared with Grade IV disease. Similar findings have been shown in other studies $[19,24]$. T-stage was also shown to be an independent prognostic factor in our study, with improved survival observed in patients with T1 stage compared with T2-TX. Hence, in relation to TNM the prognostic value in terms of survival prediction, in our study, can be based on T-stage alone. This is in alignment with ASCO guidelines [26]. Although platinum combinations with a third-generation chemotherapy agent are widely recognized as standard of care for first-line treatment of patients with advanced NSCLC, the choice of platinum agent (carboplatin or cisplatin) varies. In our study, the choice of platinum agent was shown to influence survival, with cisplatin proving to be more effective than carboplatin. This finding is consistent with a meta-analysis, in which carboplatin combinations with third-generation chemotherapy were shown to be inferior to cisplatin combinations with third-generation chemotherapy [27].
Genetic factors are also involved in lung cancer development, with aberrant promoter methylation playing an important role. Previous studies of NSCLC have reported varied frequencies of $p 16$ methylation, with Wang et al (2008) reporting $p 16$ methylation in $38 \%$ of samples [28], while Guzman et al (2007) observed $p 16$ methylation in $79.7 \%$ of samples [29]. In this study, the p16 methylation observed was low $(17.6 \%)$ compared to observations in previous studies. Additionally, RASSF1A methylation has been implicated in NSCLC, with RASSF1A methylation reported in $21 \%$ [28] and $40 \%$ [30] of samples, while in the current study RASSF1A was observed in only $8.2 \%$ of samples. Possible explanations for the different methylation frequencies include techniques to study methylation status and study population. Wang et al (2008) [28] utilized microarray technology, while Guzman et al (2007) [29], Li et al (2003) [30] and the current study utilized PCR-based technology. These studies focused on samples from China [28], Chile [29] and the US [30], respectively, whereas the current study analyzed samples from nine different countries (China, Egypt, Israel, Pakistan, Poland, Romania, South Korea, Taiwan, and Turkey). Moreover, different frequencies of methylation have been observed in different NSCLC histological types [29], thus different proportions of histological types may be present in each of the different studies.

The naturalistic setting of this study provides insight into the use of gemcitabine-platinum first-line therapy and treatment outcomes. In addition to the strengths of this study, we acknowledge several limitations which should be taken into account when interpreting the results. Firstly, as the number of patients enrolled in this study was reduced $(n=1214)$ compared with the planned number $(n=3000)$ the study was not adequately powered to test the hypothesis that smoking during chemotherapy may be associated with shorter survival in patients with advanced NSCLC who receive first-line chemotherapy with gemcitabine-platinum. Secondly, there was a higher than anticipated decrease in smoking during therapy. Thirdly, the observational nature of the study can lead to bias and confounding.

\section{Conclusions}

This observational study shows that continued smoking during gemcitabine-platinum therapy was not associated with shorter survival in patients with advanced NSCLC. Additionally, construction and validation of a predictive model identified four independent prognostic factors that were associated with survival - disease stage, performance status, gemcitabine-platinum regimen, and T-stage. 


\section{Authors' contributions}

KK conceived the study design. SA, C-TL, MMarek, SZG, YK, MMeshref and SQ acquired the data. KK, ZK, MMeshref and SA analyzed and interpreted the data. SA and MMeshref contributed to the drafting of the manuscript. C-TL, MMarek, SZG, YK, $\mathrm{SQ}, \mathrm{ZK}, \mathrm{KK}$ and SA critically revised the manuscript drafts. All authors read and approved the final manuscript.

\section{Acknowledgements}

This study was supported by a research grant from Eli Lilly (Eli Lilly and Company, Indianapolis USA). The authors would like to thank the B9E-AA-B004 study group in each country for their participation and contribution to this study, Steve Vander Hoorn for statistical support, and Trisha Dwight (Eli Lilly Australia) for medical writing support.

\section{Conflict of Interests}

Chien-Te Li, Magdalena Marek, Salih Z Guclu, Younseup Kim and Shukui Qin were investigators for trial B9E-AA-B004 and have no other competing interests. Mohamed Meshref was a clinical investigator for this trial while the trial was active and is now an employee of Eli Lilly and Company. Sedat Altug is an employee of Eli Lilly and Company. Zbigniew Kadziola and Kurt Krejcy are employees of, and hold stock/stock options in, Eli Lilly and Company.

\section{References}

1. Parkin DM, Bray F, Ferlay J, et al. Global cancer statistics, 2002. CA Cancer J Clin. 2005; 55: 74-108.

2. Pisani P, Parkin DM, Bray F, et al. Estimates of the worldwide mortality from 25 cancers in 1990. Int J Cancer. 1999; 83: 18-29.

3. Araujo A, Ribeiro R, Azevedo I,et al. Genetic polymorphisms of the epidermal growth factor and related receptor in non-small cell lung cancer-a review of the literature. Oncologist. 2007; 12: 201-210.

4. Blackhall F, Ranson $\mathrm{M}$, Thatcher N. Where next for gefitinib in patients with lung cancer? Lancet Oncol. 2006; 7: 499-507.

5. Ginsberg RJ, Vokes EE, Rosenzweig K. Non-small cell lung cancer. In: DeVita VT, et al, eds. Cancer: Principles and Practice of Oncology; 6th ed. Philadelphia: Lippincott-Raven; 2001: 925-983.

6. Hecht SS. Tobacco smoke carcinogens and lung cancer. J Natl Cancer Inst. 1999; 91: 1194-1210.

7. Hecht SS. Cigarette smoking and lung cancer: chemical mechanisms and approaches to prevention. Lancet Oncol. 2002; 3: 461-469.

8. Yuspa SH, Shields PG. Etiology of cancer: chemical factors. In: DeVita VT, et al, eds. Cancer: Principles and Practice of Oncology; 6th ed. Philadelphia: Lippincott-Raven; 2001: 179-193.

9. Finkelstein DM, Ettinger DS, Ruckdeschel JC. Long-term survivors in metastatic non-small-cell lung cancer: an Eastern Cooperative Oncology Group Study. J Clin Oncol. 1986; 4: 702-709.
10. O'Connell JP, Kris MG, Gralla RJ, et al. Frequency and prognostic importance of pretreatment clinical characteristics in patients with advanced non-small-cell lung cancer treated with combination chemotherapy. J Clin Oncol. 1986; 4: 1604-1614.

11. Albain KS, Crowley JJ, LeBlanc M, et al. Survival determinants in extensive-stage non-small-cell lung cancer: the Southwest Oncology Group experience. J Clin Oncol. 1991; 9: 1618-1626.

12. Socinski MA, Morris DE, Masters GA, et al. Chemotherapeutic management of stage IV non-small cell lung cancer. Chest. 2003; 123 (1 Suppl): 226S-243S.

13. Gregorc V, Ludovini V, Pistola L, et al. Relevance of p53, bcl-2 and $\mathrm{Rb}$ expression on resistance to cisplatin-based chemotherapy in advanced non-small cell lung cancer. Lung Cancer. 2003; 39: 41-48.

14. Murakami I, Hiyama K, Ishioka S, et al. p53 gene mutations are associated with shortened survival in patients with advanced non-small cell lung cancer: an analysis of medically managed patients. Clin Cancer Res. 2000; 6: 526-530.

15. Rosell R, Fossella F, Milas L. Molecular markers and targeted therapy with novel agents: prospects in the treatment of non-small cell lung cancer. Lung Cancer. 2002; 38 (Suppl 4): 43-49.

16. van Houwelingen HC. Validation, calibration, revision and combination of prognostic survival models. Stat Med. 2000; 19: 3401-3415.

17. Brundage MD, Davies D, Mackillop WJ. Prognostic factors in non-small cell lung cancer: a decade of progress. Chest. 2002; 122: 1037-1057.

18. Ahuja R, Weibel SB, Leone FT. Lung cancer: the oncologist's role in smoking cessation. Semin Oncol. 2003; 30: 94-103.

19. Toh CK, Wong EH, Lim WT, et al. The impact of smoking status on the behavior and survival outcome of patients with advanced non-small cell lung cancer: a retrospective analysis. Chest. 2004; 126: 1750-1756.

20. Tsao AS, Liu D, Lee JJ, et al. Smoking affects treatment outcome in patients with advanced nonsmall cell lung cancer. Cancer. 2006; 106(11): 2428-2436.

21. Scagliotti GV, Parikh P, Von Pawel J, et al. Phase III study comparing cisplatin plus gemcitabine with cisplatin plus pemetrexed in chemotherapy-naive patients with advanced-stage non-small-cell lung cancer. J Clin Oncol. 2008; 26: 3543-3551.

22. Nguyen B, Paul S, Posther K, et al. The effect of smoking history on survival outcome: An exploratory analysis of a phase III study of gemcitabine and cisplatin in patients with advanced non-small cell lung cancer (NSCLC). J Clin Oncol. 2006; 24(18S): Abstract7141.

23. Hoang $\mathrm{T}, \mathrm{Xu} \mathrm{R}$, Schiller JH, et al. Clinical model to predict survival in chemonaive patients with advanced non-small-cell lung cancer treated with third-generation chemotherapy regimens based on eastern cooperative oncology group data. J Clin Oncol. 2005; 23: 175-183.

24. Mandrekar SJ, Schild SE, Hillman SL, et al. A prognostic model for advanced stage nonsmall cell lung cancer. Pooled analysis of North Central Cancer Treatment Group trials. Cancer. 2006; 107: 781-792.

25. Paesmans M, Sculier JP, Libert P, et al. Prognostic factors for survival in advanced non-small-cell lung cancer: univariate and multivariate analyses including recursive partitioning and amalgamation algorithms in 1,052 patients. The European Lung Cancer Working Party. J Clin Oncol. 1995; 13: 1221-1230.

26. Pfister DG, Johnson DH, Azzoli CG, et al. American Society of Clinical Oncology treatment of unresectable non-small-cell lung cancer guideline: update 2003. J Clin Oncol. 2004; 22: 330-353.

27. Ardizzoni A, Boni L, Tiseo M, et al. Cisplatin- versus carboplatin-based chemotherapy in first-line treatment of advanced non-small-cell lung cancer: an individual patient data meta-analysis. J Natl Cancer Inst. 2007; 99: 847-857. 
28. Wang Y, Zhang D, Zheng W, et al. Multiple gene methylation of nonsmall cell lung cancers evaluated with 3-dimensional microarray. Cancer. 2008; 112: 1325-1336.

29. Guzman LM, Koriyama C, Akiba S, et al. High frequency of p16 promoter methylation in non-small cell lung carcinomas from Chile. Biol Res. 2007; 40: 365-372.

30. Li J, Zhang Z, Dai Z, et al. RASSF1A promoter methylation and Kras2 mutations in non small cell lung cancer. Neoplasia. 2003; 5: 362-366. 